\title{
The Possibility of a Disk Formation around Late B Stars
}

\author{
Jiří Kubát ${ }^{1}$, Jiří Krtička ${ }^{2,3,1}$, Izold B. Pustylnik ${ }^{4}$, and Viktor Votruba ${ }^{3,1}$ \\ ${ }^{1}$ Astronomický ústav $A V \check{C} R, C Z$-25165 Ondřejov, Czech Republic \\ ${ }^{2}$ Department of Physics and Astronomy, University of Glasgow, \\ Glasgow G12 8QQ, UK \\ ${ }^{3}$ Ústav teoretické fyziky a astrofyziky PřF MU, Kotlářská 2, CZ-611 37 \\ Brno, Czech Republic \\ ${ }^{4}$ Tartu Observatory, 61602 Tõravere, Estonia
}

\begin{abstract}
Line driven wind in late B stars causes significant heating of layers just above the photosphere. This large heating is a source of a thermal wind, which together with fast rotation opens the possibility of a formation of a disk. We refer to such disk as to a radiation induced disk. Disk inhibition by nonradial line force has only little effect in this case, since absorption in spectral lines is not the main wind driving mechanism.
\end{abstract}

\section{Introduction}

Cool Be stars form a special subclass of Be stars with several characteristic features. They often display more or less stable strong emission hydrogen Balmer lines and no rapid variations (thus excluding the interesting phenomenon of nonradial pulsations). A large number of them also does not show any sign of an orbital motion or the evidence of the mass transfer in a binary system is very weak, so it is evident that the source of the Balmer emission is not the binarity with corresponding flows, but it must be somewhere else.

Krtička \& Kubát (2001a, 2001b) found significant heating in the multicomponent radiatively driven winds of stars of the spectral type B4 and cooler. Heating for these stellar types is caused basically by friction, which may heat the medium to temperatures of the order of million degrees and form a region which may be called as a corona. Such corona may be a source of another type of a wind, namely the coronal wind driven by a thermal pressure. Kubát et al. (2003) modeled such wind as a three-component stellar wind consisting of hydrogen, electrons, and metallic ions with assumed fixed temperature distribution taken in the lower part from Figure 8 in Krtička \& Kubát (2002b) and in the upper part approximated by an expression $T(r)=T_{0}\left(r_{0} / r\right)^{2 / 7}$. The latter expression follows from the equation of thermal conduction (Parker 1958). Here, $r_{0}$ and $T_{0}$ are radius and temperature at the base of the coronal wind.

This wind is driven by a thermal pressure and replaces the original radiatively driven wind. Since the mechanism of the driving of such wind is the high temperature of the corona, which is obtained by friction caused by the velocity difference induced by radiative driving, we denote such wind as a radiation induced coronal wind. 


\section{The Possibility of a Disk Formation}

The mechanism of formation of a disk around single Be stars is still unknown. The influence of rotation on a radiatively driven stellar wind was studied by a number of authors. The interesting model of a line driven wind compressed disk was introduced by Bjorkman \& Cassinelli (1993), then it was confirmed by numerical simulations of Owocki et al. (1994), and later numerical investigation lead to the conclusion that the formation of a wind compressed disk is inhibited by the nonradial component of the radiative force (Owocki et al. 1996).

However, we suggest a mechanism of a disk formation based on this idea of a wind compressed disk. First, large heating caused by friction in a multicomponent wind changes the ionization structure of the wind and switches off the radiative driving. Large temperature switches on the thermal pressure driving. The disk inhibition by a radiation force does not work for pressure driven wind and a disk similar to the wind compressed disk may be formed. Such disk we call as a radiation induced disk here. However, there is also a weak point in this suggested mechanism, which lies mainly in the fact that coronal winds give usually low mass loss rates. This may cause troubles for a formation of a disk within a reasonable time interval. However, the answer to these doubts may be given by hydrodynamical simulations, which will be done in near future.

\section{Summary}

In this paper we suggested a mechanism of a formation of a disk around cool $\mathrm{B}$ stars based on a combination of a radiative driving mechanism (which is relatively weak and causes basically heating of the medium), a thermal driving mechanism which exploits the result of a heating (a large temperature) producing the stellar wind, and of a compression of the wind towards the equatorial plane. The disk inhibition has not effect since the radiative force is negligible. However, the possibility of a formation of a wind must be verified, at best by detailed hydrodynamical calculations. These simulations may give answer to the question, if the ionic component of a radiatively driven wind follows the passive component one and forms a disk or produces a separate polar wind. These calculations are in progress.

Acknowledgments. This work was supported by grants 205/02/0445 and 205/01/0656 (GA ČR), by projects K2043105 and AV 0Z1 003909, and by PPARC Rolling Grant. Participation at the meeting was supported by an IAU grant.

\section{References}

Bjorkman, J. E., Cassinelli, J. P. 1993, ApJ, 409, 429

Krtička, J., Kubát, J. 2001a, A\&A, 369, 222

Krtička, J., Kubát, J. 2001b, A\&A, 377, 175

Kubát, J., Krtička, J., Pustylnik, I. B. 2003, A\&A, submitted

Owocki, S. P., Cranmer, S. R., Blondin, J. M. 1994, ApJ, 424, 887

Owocki, S. P., Cranmer, S. R., Gayley, K. G. 1996, ApJ, 472, L115

Parker, E. N. 1958, ApJ, 128, 664 